\title{
Partición de la precipitación en un bosque tropical montano de pino-encino en el centro de México
}

\author{
Precipitation partition in a tropical montane pine-oak forest in central Mexico
}

\begin{abstract}
Alberto Gómez-Tagle Ch a*, A Francisco Gómez-Tagle R a , J Alejandro Ávila O a , Leendert Adrian Bruijnzeel ${ }^{\text {b }}$
*Autor de correspondencia: ${ }^{a}$ Universidad Michoacana de San Nicolás de Hidalgo, Instituto de Investigaciones sobre los Recursos Naturales, Departamento de Ciencias de la Tierra, Morelia, México, Av. San Juanito Itzícuaro SN, C.P. 58330, Morelia, Michoacán, México, tel.: 52-443-3272350 (ext.109), fax: 52-443-3272351, algomez@umich.mx

b Vrije Universiteit Amsterdam, Faculty of Earth and Life Sciences, Critical Zone Group, The Netherlands.
\end{abstract}

\begin{abstract}
SUMMARY
Precipitation partitioning is a key process in the terrestrial phase of the water cycle since it regulates the amount of water available for other processes. Precipitation partitioning in throughfall, stemflow and interception loss was measured on a daily basis in a tropical pine-oak forest at 2,160 m elevation in the Cuitzeo watershed, located in central Mexico for 26 months. The recorded precipitation of 2,882 mm (Aug.28/2010 - Oct.23/2013) included 242 events; $35.5 \%$ of rainfall events were $<5$ mm and only $16.1 \%$ exceeded $20 \mathrm{~mm}$. Throughfall $(T F)$, stemflow $(S F)$ and interception loss $\left(E_{i}\right)$ corresponded to $80.6 \%, 2.4 \%$ and $17.2 \%$ of gross precipitation $\left(P_{g}\right)$, respectively. Free $T F$ component $(p)$ was $0.86 \mathrm{~mm} \mathrm{~mm}^{-1}$ and canopy storage capacity $(S) 0.29 \mathrm{~mm}$. Trunk storage capacity $\left(S_{t}\right)$ averaged $0.023 \mathrm{~mm}$ and mean stemflow proportion $\left(p_{t}\right)$ was $0.0055 \mathrm{~mm} \mathrm{~mm}^{-1}$. SF index averaged $0.411 \mathrm{~L} \mathrm{~mm}^{-1}$ per tree while mean trunk capacity volume was 1.43 L. $E_{i}$ correlated negatively with $P_{g}$ while $T F$ correlated positively with $P_{g}$. Empirical models that describe $S F$ index (as volume) and trunk capacity volume as functions of tree height, tree diameter at breast height and projected crown surface were successfully fitted.
\end{abstract}

Key words: stemflow, throughfall, stemflow index, canopy storage capacity.

\section{RESUMEN}

La partición de la precipitación es clave en la fase terrestre del ciclo hidrológico pues determina el agua disponible para otros procesos. En este estudio se midió diariamente durante 26 meses (28/08/2010 - 23/10/2013) la partición de la precipitación en precipitación directa, escorrentía cortical e interceptación en un bosque tropical de pino-encino a $2.160 \mathrm{~m}$ de elevación en el sur de la cuenca del Lago de Cuitzeo, centro de México. La precipitación total medida fue de $2.882 \mathrm{~mm}$ en 242 eventos. El 35,5 \% de los eventos de precipitación tuvieron láminas $<5 \mathrm{~mm}$ y solo el 16,1 \% excedió $20 \mathrm{~mm}$. La precipitación directa, la escorrentía cortical y la pérdida por interceptación correspondieron a $80,6 \%, 2,4 \%$ y $17,2 \%$ de la precipitación bruta $\left(P_{g}\right)$, respectivamente. La precipitación directa libre $(p)$ fue de $0,86 \mathrm{~mm} \mathrm{~mm}^{-1}$ y la capacidad de retención del dosel $(S)$ 0,29 $\mathrm{mm}$. El promedio de la capacidad de almacenamiento del tronco $\left(S_{t}\right)$ fue $0,023 \mathrm{~mm}$ y la proporción de precipitación que alcanza las ramas y se convierte en escorrentía cortical $\left(p_{t}\right) 0,0055 \mathrm{~mm}$ $\mathrm{mm}^{-1}$. El índice de escorrentía cortical medio fue de $0,411 \mathrm{~L} \mathrm{~mm}^{-1}$ de lluvia mientras que el volumen de almacenamiento promedio de 1,43 L. La pérdida por interceptación correlacionó negativamente con $P_{g}$ mientras que la precipitación mostró una relación positiva. Se generaron modelos empíricos empleando la altura del árbol, el diámetro normal y la superficie de copa como predictores del índice de escorrentía cortical y el volumen de almacenamiento.

Palabras clave: escorrentía cortical, precipitación bajo dosel, índice de escorrentía cortical, capacidad de retención del dosel.

\section{INTRODUCCIÓN}

Los bosques tienen un papel esencial en los ciclos del agua, nutrientes y carbono a escala global. Desde una perspectiva hidrológica, los bosques funcionan como reserva de agua, regulan su flujo y evitan la erosión del suelo, además provén servicios y bienes como la madera, agua potable o posibilidades recreativas (Nahuelhual et al. 2007). En México la tasa de deforestación y cambio de uso se ha reducido de forma importante en las últimas décadas. FAO
(2011) en el periodo 1990-2000 reporta para México una tasa de deforestación anual de $-0,52 \%$ que se redujo a - $0,3 \%$ para el periodo 2000-2010.

En el centro de México, las zonas boscosas son áreas de captación hídrica de las cuencas que abastecen centros de población en donde habitan aproximadamente 42,7 millones de personas (INEGI 2013). Peñuela y Carrillo (2013) emplearon la teoría de los sistemas de flujo y correlacionaron indicadores superficiales para definir zonas de recarga y descarga de agua subterránea en la porción 
centro sur de la Mesa Central en México y mencionan que la presencia de bosques de Pinus spp. y bosques de encino (Quercus spp.) están relacionados con zonas de recarga.

La partición de la precipitación por la vegetación tiene un papel clave en la fase terrestre del ciclo hidrológico. Durante un evento de precipitación, una parte de la precipitación bruta $\left(P_{g}\right)^{l}$ puede alcanzar el suelo como precipitación directa $(T F)$. Cuando entra por los espacios del dosel se le denomina precipitación directa libre $(p)$. Otra parte de la precipitación bruta alcanza las ramas (fracción de escorrentía cortical; $p_{t}$ ), satura los troncos de los árboles (capacidad de almacenamiento del tronco; $S_{t}$ ) y fluye hasta alcanzar el suelo como escorrentía cortical $(S F)$. El agua restante que llega al dosel $\left(1-p-p_{t}\right)$ y es retenida por este hasta alcanzar la capacidad de retención o saturación del dosel $(S)$ puede gotear para sumarse a la precipitación directa o se evapora parcialmente durante y después del evento de precipitación denominándose entonces pérdida por interceptación $\left(E_{i}\right)$ (Gash y Morton 1978, David et al. 2005).

Se ha estudiado la partición de la precipitación en diversos tipos de vegetación y ecosistemas; desde ambientes áridos y semiáridos (Martínez-Meza y Withford 1996, Návar et al. 1999, Carlyle-Moses 2004) hasta bosques tropicales (Hölscher et al. 2004), bosques de niebla ${ }^{2}$ (Holwerda et al. 2010) y bosques templados (Rutter et al. 1975, Návar 2011). Estudios recientes abordan el tema con una perspectiva química y de balance de nutrientes (Levia y Frost 2003). Otros analizan la partición de la precipitación en sus componentes y la variación temporal e interespecífica de la interceptación y la escorrentía cortical (Návar 2011) y algunos se han centrado en la importancia de la escorrentía cortical en la redistribución del agua y sus implicaciones ecohidrológicas (Levia y Frost 2003, Holwerda et al. 2010, Návar 2011).

Para el caso de México, los estudios de partición de la precipitación son relativamente escasos y se concentran en cinco tipos de ambientes; bosques de niebla en la vertiente atlántica (Holwerda et al. 2010, 2013), vegetación semiárida del centro (matorral inerme espinoso) (Flores et al. 2013), norte y noreste (Návar et al. 1999, Carlyle-Moses 2004, Cantú y González 2005, Návar 2011), bosques templados del noreste (Charles 1998, Cantú y González 2002, Carlyle-Moses y Price 2007), plantaciones de pino y bosques de encino del centro de México (Michoacán) (DíazFernández y Gómez-Tagle 2012).

Debido a la relevancia hidrológica de los bosques del centro de México, es necesario conocer y describir adecuadamente la partición de la precipitación para contar con información detallada de algunos de los procesos del

En este trabajo se utilizan las abreviaturas y simbología para los diferentes componentes de la partición de la precipitación y el proceso de interceptación siguiendo la nomenclatura de David et al. (2005).

2 En este trabajo se utiliza el término "bosque de niebla", aunque en México este tipo de formación vegetal es llamado "bosque mesófilo" siguiendo la clasificación de Rzedowski (2006). ciclo del agua, fundamental para llevar a cabo un adecuado ordenamiento hidrológico. El presente trabajo tiene una doble justificación; a) generar conocimiento científico sobre el funcionamiento hidrológico de los bosques de pinoencino en el centro de México y b) proveer información de referencia sobre la partición de la precipitación en un sitio dentro de la zona de recarga de los mantos freáticos de la ciudad de Morelia, Michoacán.

Por lo tanto, los objetivos de este trabajo son: (1) cuantificar la partición de la precipitación en precipitación directa, escurrimiento caulinar y calcular mediante la diferencia la pérdida de lluvia por interceptación (I); y (2) investigar si existen relaciones entre la generación de escorrentía cortical y las características dasométricas de los árboles, incluyendo diámetro normal, altura y superficie de copa.

\section{MÉTODOS}

Área de estudio. El estudio se realizó en la estación de investigación ecohidrológica Alto Fresno $\left(19^{\circ} 33^{\prime} 00^{\prime \prime} \mathrm{N}\right.$, $101^{\circ} 14^{\prime} 24^{\prime \prime}$ O) en la parte alta del sur de la cuenca del Lago de Cuitzeo, ubicada a $18 \mathrm{~km}$ al SSO de la ciudad de Morelia, Michoacán en el centro de México (figura 1). De acuerdo con Garduño et al. (2014) el área de estudio se encuentra dentro de la zona de recarga más importante de los mantos freáticos de la ciudad de Morelia.

Las mediciones de campo fueron hechas en una zona resguardada con cerca perimetral con superficie de 2,5 ha que forma parte de la cuenca 1 (11,7 ha) de la estación ecohidrológica. El sitio se encuentra a los 2.160 m.s.n.m., tiene una exposición SSO y una pendiente media de $13,4^{\circ}$. La comunidad vegetal es un bosque secundario de pinoencino con edad promedio de 20 años, pero con individuos que alcanzan los 45 años. Las principales especies del estrato arbóreo son: Pinus devoniana Lindl., P. douglasiana Martínez, P. leoiphylla Schiede, además de encinos Quercus castanea Neé y Q. obtusata Bonpl.

El clima es templado subhúmedo con precipitaciones de verano (>95\%) normalmente de junio a octubre y baja precipitación invernal $(<5 \%)$ (diciembre - febrero). El Servicio Meteorológico Nacional (SMN) reporta para las estaciones más cercanas: 16001 Acuitzio del Canje (2.200 m s.n.m.) y 16055 Jesús del Monte (2.180 m s.n.m.) temperaturas promedio anuales de 16,9 y $17,5^{\circ} \mathrm{C}$ y precipitaciones promedio anuales de $1.115,8$ y $953,9 \mathrm{~mm}$, respectivamente, en el periodo 1951-2010 (SMN 2011ab).

Los suelos son de origen volcánico, el epipedón corresponde a un Cambisol, mientras que el endopedón corresponde a un suelo sepultado con un horizonte diacrítico argílico, en este caso un Lixisol húmico (Gómez-Tagle 2008).

Caracterización dasométrica. Se realizó una caracterización dasométrica del bosque empleando seis sitios circulares de dimensiones fijas de $12 \mathrm{~m}$ de diámetro $\left(113,1 \mathrm{~m}^{2}\right.$ cada uno). Se registraron para todos los individuos con 
diámetro normal (a 1,3 m) > $10 \mathrm{~cm}$; especie, diámetro, altura total y superficie de copa. Para los pinos se registró la edad usando núcleos obtenidos mediante un taladro de Pressler.

Precipitación. Para la cuantificación de la precipitación bruta $\left(P_{g}\right)$ se empleó un pluviómetro manual con diámetro de $34 \mathrm{~cm}\left(907,9 \mathrm{~cm}^{2}\right)$ y una estación meteorológica automática Davis Vantage Pro2 con pluviómetro de balancín. Estos se ubicaron a 1,5 y 1,8 m sobre el nivel del suelo en un área despejada a una distancia de aproximada $250 \mathrm{~m}$ de la zona instrumentada y a $50 \mathrm{~m}$ una de otra siguiendo los criterios de Carlyle-Moses (2004). La lámina del pluviómetro manual se obtuvo dividiendo el volumen colectado entre la superficie del pluviómetro. La estación automática fue calibrada y ajustada para lograr una resolución de 0,2 mm siguiendo la metodología de Calder y Kidd (1978) y se programó para realizar registros cada $10 \mathrm{~min}$. Estos posteriormente se sumaron para obtener valores cada 24 horas. Los registros del pluviómetro manual se realizaron cada 24 horas entre las 8:00 y 9:00 am de hora local. El promedio de lámina de precipitación diaria se obtuvo empleando los registros de la estación meteorológica y del pluviómetro manual.

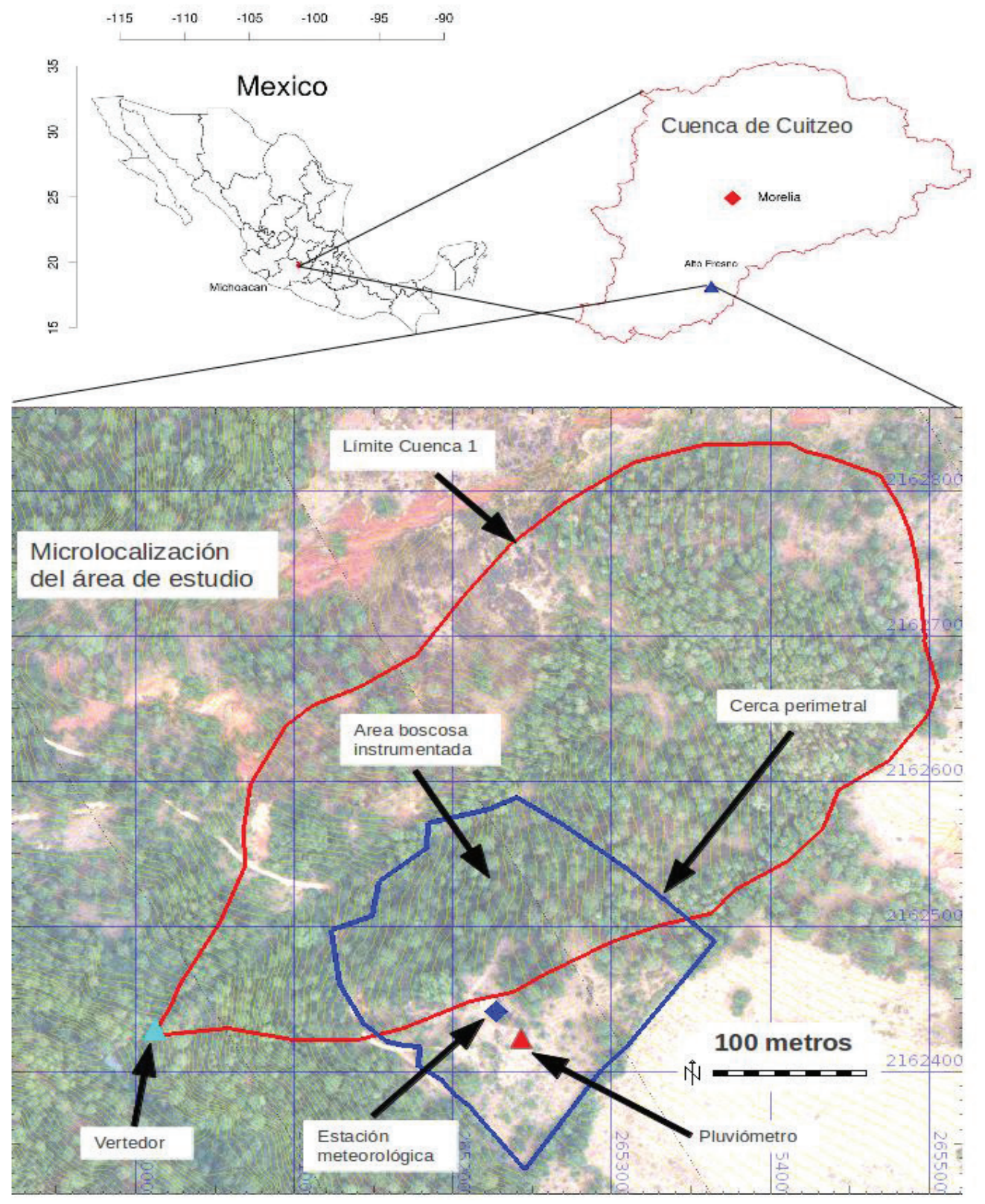

Figura 1. Ubicación del área de estudio, estación ecohidrológica Alto Fresno en el centro de México.

Location of the Alto Fresno Ecohydrological Research Station, Central Mexico. 
Precipitación directa (TF). Se determinó empleando seis canaletas de acero galvanizado con sección en "V" con dimensiones de $1,0 \times 0,25 \mathrm{~m}\left(0,25 \mathrm{~m}^{2}\right.$ de superficie), abertura de $62^{\circ}$, altura de $20 \mathrm{~cm}$ y una pestaña de $4,5 \mathrm{~cm}$ en el margen superior para evitar pérdidas por salpicadura. Las canaletas se instalaron con un ángulo de $15^{\circ}$ en soportes de madera a 1,0 $\mathrm{m}$ de altura sobre el suelo y se conectaron mediante tubos de PVC a recipientes de almacenamiento (20,0 L) con tapa. Las canaletas se reubicaron aleatoriamente dentro del sitio cada 15 días de acuerdo con lo recomendado por Holwerda et al. (2006).

Escorrentía cortical (SF). Se midió en 22 árboles seleccionados al azar, 15 pinos y siete encinos (cuadro 1). Cada uno de los troncos fue equipado con un sistema colector fabricado de manguera plástica PVC y conectado a un recipiente de almacenamiento con tapa. El anillo colector se fijó al árbol con clavos en una sección del tronco con la corteza previamente alisada, y posteriormente se selló a la corteza empleando silicón industrial. Para cada árbol se registraron por lo menos 80 días con precipitación bruta $>$ $0,01 \mathrm{~mm}$.

Los registros de precipitación directa y escorrentía cortical se realizaron en forma manual cada 24 horas entre las 8:00 y 9:00 am de hora local, con una precisión de $\pm 5,0$ $\mathrm{mL}$. El intervalo de registro abarcó del 28 de agosto de 2010 al 23 de octubre de 2013. Debido a que los registros se realizaron cada 24 horas, en este trabajo se emplea el término evento para referirse a la precipitación acumulada en un periodo de 24 horas de acuerdo con el criterio de Gash et al. (1980).

Los valores de escorrentía cortical registrados en unidades volumétricas $\left(\mathrm{L} ; S F_{V}\right)$ se convirtieron a lámina $(\mathrm{mm}$; $S F_{L}$ ) para cada árbol y evento dividiendo el valor de $S F_{V}$ entre la superficie de copa proyectada del árbol en metros cuadrados. Para ciertas fechas en el periodo de estudio ocurrió derramamiento de algunos de los recipientes de recolección y fueron excluidos del análisis.

Cuadro 1. Características estructurales de los árboles usados en las mediciones de escorrentía cortical y los periodos de registro de datos. Structural characteristics of trees used for stemflow $(S F)$ measurements and the data collection period.

\begin{tabular}{clcccccc}
\hline \multirow{2}{*}{ Árbol } & Especie & Diámetro $(\mathrm{cm})$ & Altura $(\mathrm{m})$ & $\begin{array}{c}\text { Superficie de } \\
\text { copa }\left(\mathrm{m}^{2}\right)\end{array}$ & Edad (años) & \multicolumn{2}{c}{ Periodo de registro } \\
\cline { 6 - 8 } P1 & Pinus devoniana & 28,0 & 17,4 & 229,26 & 21 & $02 / 09 / 10$ & $05 / 07 / 12$ \\
P2 & P. devoniana & 41,4 & 18,2 & 516,73 & 20 & $16 / 07 / 11$ & $23 / 10 / 13$ \\
P3 & P. devoniana & 26,4 & 13,2 & 277,59 & 14 & $16 / 07 / 11$ & $01 / 09 / 12$ \\
P4 & P. devoniana & 30,9 & 12,7 & 176,71 & 12 & $16 / 07 / 11$ & $23 / 10 / 13$ \\
P5 & P. devoniana & 22,6 & 9,5 & 153,94 & 11 & $16 / 07 / 11$ & $01 / 09 / 12$ \\
P6 & P. devoniana & 37,9 & 15,2 & 326,85 & 15 & $16 / 07 / 11$ & $01 / 09 / 12$ \\
P7 & P. leiophylla & 39,5 & 17,1 & 300,95 & 16 & $17 / 07 / 11$ & $29 / 08 / 12$ \\
P8 & P. devoniana & 13,7 & 10,5 & 80,12 & 12 & $17 / 07 / 11$ & $01 / 09 / 12$ \\
P9 & P. devoniana & 35,6 & 20,0 & 402,93 & 17 & $13 / 09 / 11$ & $01 / 09 / 12$ \\
P10 & P. leiophylla & 48,4 & 19,6 & 559,90 & 24 & $12 / 09 / 11$ & $01 / 09 / 12$ \\
P11 & P. devoniana & 33,1 & 18,2 & 428,22 & 18 & $13 / 09 / 11$ & $01 / 09 / 12$ \\
P12 & P. leiophylla & 78,7 & 30,3 & 312,90 & 41 & $05 / 09 / 12$ & $23 / 10 / 13$ \\
P13 & P. leiophylla & 29,9 & 22,2 & 73,90 & 18 & $05 / 09 / 12$ & $23 / 10 / 13$ \\
P14 & P. leiophylla & 22,4 & 17,9 & 32,17 & 14 & $05 / 09 / 12$ & $23 / 10 / 13$ \\
P15 & P. leiophylla & 33,2 & 22,2 & 11,95 & 16 & $05 / 09 / 12$ & $23 / 10 / 13$ \\
Q1 & Quercus castanea & 24,5 & 9,8 & 40,72 & - & $13 / 09 / 11$ & $23 / 10 / 13$ \\
Q2 & Q. castanea & 29,9 & 8,6 & 53,33 & - & $13 / 09 / 11$ & $23 / 10 / 13$ \\
Q3 & Q. castanea & 28,3 & 9,4 & 47,78 & - & $06 / 07 / 12$ & $23 / 10 / 13$ \\
Q4 & Q. castanea & 14,1 & 8,5 & 22,23 & - & $05 / 09 / 12$ & $23 / 10 / 13$ \\
Q5 & Q. obtusata & 26,4 & 9,8 & 51,78 & - & $05 / 09 / 12$ & $23 / 10 / 13$ \\
Q6 & Q. obtusata & 31,7 & 9,4 & 49,02 & - & $05 / 09 / 12$ & $23 / 10 / 13$ \\
Q7 & Q. obtusata & 39,4 & 7,1 & 84,30 & - & $05 / 09 / 12$ & $23 / 10 / 13$ \\
\hline
\end{tabular}


Partición de la precipitación. Primeramente se obtuvieron la capacidad de retención del dosel $(S)$ y el coeficiente de precipitación directa libre $(p)$, siguiendo la aproximación de Leyton et al. (1967) a partir de la relación $P_{g} v s . T F$ :

$$
T F=p^{*} P_{g}-S
$$

Donde, $T F=$ precipitación directa;

$p=$ pendiente de la recta; $P_{g}=$ precipitación bruta; $S=$ valor negativo del intercepto.

La capacidad de almacenamiento del tronco $\left(S_{t}\right)$ y la proporción de $P_{g}$ que llega a las ramas y al tronco y se traduce en escorrentía cortical $\left(p_{t}\right)$ se obtuvieron empleando el método de Gash y Morton (1978) utilizando la siguiente expresión:

$$
S F_{L j}=p_{t j} * P_{g}-S_{t j}
$$

Donde,

$S F_{L j}=$ escorrentía cortical en lámina $(\mathrm{mm})$ para el árbol $j$.

$p_{t j}=$ fracción de escorrentía cortical; pendiente de la recta para el árbol $j$.

$S_{t j}=$ capacidad de almacenamiento del tronco; intercepto negativo de la recta para el árbol $j$.

Además se obtuvieron el índice de escorrentía cortical (Iec) y el volumen de almacenamiento del tronco $(V)$ empleando la siguiente expresión:

$$
S F_{V j}=I e c_{j} * P_{g}+V_{t j}
$$

Donde,

$S F_{V j}=$ escorrentía cortical en volumen (L) para el árbol $j$.

$I e c=$ pendiente de la recta para el árbol $j$.

$V_{t}=$ intercepto negativo de la recta para el árbol $j$.

El índice de escorrentía cortical es análogo a $p_{t}$ pero en unidades de volumen por milímetro de precipitación, mientras que volumen de almacenamiento del tronco es análogo a $S_{t}$ pero en unidades de volumen. En este sentido $S_{t}$ representa el volumen de retención de agua del árbol $j$ antes de generar escorrentía cortical.

La lámina de interceptación se determinó empleando la expresión:

$$
I=P_{g}-T F
$$

Mientras que la interceptación como proporción de la precipitación bruta $\left(P_{g}\right)$ se calculó a partir de la siguiente expresión:

$$
I \%=\left(I / P_{g}\right) * 100
$$

Donde,

$I \%=$ proporción de la $P_{g}$ interceptada por el dosel arbóreo en unidades porcentuales.
La precipitación neta $\left(P_{n}\right)$ se obtuvo empleando la siguiente expresión:

$$
P_{n}=T F+S F_{\text {sitio }}
$$

Donde,

$S F_{\text {sitio }}=$ proporción de $P_{g}$ correspondiente a la escorrentía cortical escalada para el sitio. Y se obtuvo a partir de los promedios de $S F_{V}$ para cada género y multiplicando por la cantidad de árboles de dicho género aplicando la siguiente expresión:

$$
S F_{g}=n_{g d} *\left(\Sigma\left(S F_{V T j} / P_{g T j}\right)\right) / n_{j}
$$

$S F_{g}=$ escorrentía cortical en $\mathrm{L} \mathrm{mm}^{-1}$ para cada género (Pinus, Quercus).

$S F_{V T j}=$ volumen de escurrimiento cortical total para el árbol $j$.

$P_{g T j}=$ lámina de precipitación total del periodo de medición del árbol $j$.

$n_{j}=$ número de árboles de cada género con medición de escurrimiento cortical.

$n_{g d}=$ número de árboles de cada género en el muestreo dasométrico.

Para el resto de los árboles (Prunus sp., Fraxinus sp., Crataegus sp.) se tomó como valor promedio $S f_{g}$ para pinos $\mathrm{y}$ encinos multiplicado por 0,5 . Se considerố que estos géneros al formar parte del segundo estrato del bosque captan menor cantidad de agua.

De forma que $S F_{\text {sitio }}$ se obtuvo aplicando la siguiente expresión:

$$
S F_{\text {sitio }}=100 *\left(\left(P_{g T s} * \Sigma S F_{g}\right) /\left(P_{g T s} * A_{m d}\right)\right)
$$

Donde,

$P_{g T s}=$ precipitación total $(\mathrm{mm})$ del periodo de estudio. $A_{m d}=$ superficie total del muestreo dasométrico.

La proporción de precipitación bruta que fluye a través del dosel $\left(T F / P_{g}\right)$ se calculó de acuerdo con el método propuesto por Carlyle-Moses (2004). Mientras que la pérdida por interceptación $\left(E_{i}\right)$ para cada evento de precipitación se obtuvo empleando la siguiente expresión:

$$
E_{i}=P_{g}-\left(T F+S F_{p}\right)
$$

Donde,

$E_{i}=$ pérdida por interceptación $(\mathrm{mm})$.

Análisis estadístico. Los análisis estadísticos se realizaron empleando el ambiente de programación estadístico $\mathrm{R} \mathrm{v}$ 3.1.1 (R Core Team 2014). Todos los parámetros de los modelos derivados en este estudio se basaron en análisis de residuales y bondad de ajuste. Los niveles de significancia, incluyendo los errores estándar asociados con los parámetros de los modelos corresponden al intervalo de confianza del $95 \%(\alpha=0,95$ o $P<0,05)$. 


\section{RESULTADOS}

Caracterización dasométrica. La densidad promedio de árboles fue de 678 individuos ha ${ }^{-1}$ con dominancia del género Pinus en el estrato arbóreo con $66,7 \%$ seguido por los Quercus spp. 17,7\%. Mientras que el 15,6\% restante comprendió tres especies; tejocote (Crataeus mexicana Moc. Sessé), capulín o cereza negra (Prunus serotina var. capuli Cav.) y fresno (Fraxinus udehi (Wenz.) Ligelsh) (cuadro 2).

Precipitación. El registro comprendió un total de 242 eventos con una lámina de precipitación acumulada de $2.881,8 \mathrm{~mm}$. El registro comprendió cuatro temporadas de lluvias (2010-2013) (figura 2). El evento de mayor precipitación $(78,1 \mathrm{~mm})$ se registró el 16 de septiembre de 2013 y correspondió a la confluencia de los huracanes Ingrid y Manuel.

La relación entre precipitación bruta medida manual y automáticamente tuvo una pendiente de $0,94 \mathrm{~mm} / \mathrm{mm}$, muy cercana a la relación 1:1 que se esperaba (figura $3 \mathrm{~A}$ ) y con $\mathrm{r}^{2}$ $=0,93(P<0,001)$. La distribución de la precipitación bruta fue fuertemente acusada y asimétrica con curtosis de 6,05 y asimetría de 2,14 . A escala de evento de lluvia, la precipitación bruta media fue de 11,9 mm con desviación estándar de 12,3 y valores mínimo y máximo de 0,01 y $78,1 \mathrm{~mm}$, respectivamente y moda de $2,5 \mathrm{~mm}$. La distribución indica que la mayoría de los eventos son pequeños, mientras que los eventos de mayor tamaño son pocos y solamente una lluvia tuvo una lámina superior a los $70 \mathrm{~mm}$ (figura 3B).

Precipitación directa (TF). Del total de eventos, 232 generaron precipitación directa con una lámina promedio acumulada de 2.323,1 $\pm 3,27 \mathrm{~mm}$. La precipitación directa mostró una fuerte correlación positiva con la precipitación bruta (coeficiente de correlación de Pearson) $\mathrm{r}=0,976(\mathrm{t}=$ $73,73, \mathrm{gl}=237, P<0,001)$.
Cuadro 2. Características dasométricas generales del bosque en la estación de investigación ecohidrológica Alto Fresno.

General structural characteristics of the forest at Alto Fresno ecohydrological research station.

\begin{tabular}{lc}
\hline Descriptor & Media $\pm \mathrm{SD}$ \\
\hline Área basal $\left(\mathrm{m}^{2} \mathrm{ha}^{-1}\right)$ & $39,7 \pm 1,43$ \\
Índice de área foliar $\left(\mathrm{m}^{2} \mathrm{~m}^{-2}\right)^{*}$ & $1,6 \pm 0,9$ \\
Altura $(\mathrm{m})$ & $12,3 \pm 7,2$ \\
Diámetro normal $(\mathrm{cm})$ & $21,3 \pm 17,4$ \\
Edad & $17,9 \pm 3,1$ \\
Superficie de copa $\left(\mathrm{m}^{2}\right)$ & $46,1 \pm 93,3$ \\
Árboles ha & $677,8 \pm 348$
\end{tabular}

SD: Desviación estándar. *Estimación mediante método semidestructivo $(\mathrm{n}=76$ ramas) provenientes de 16 individuos y regresiones alométricas, solo para Pinus sp.

La precipitación directa libre $(p)$ y la capacidad de retención del dosel $(S)$ se estimaron en $0,859 \mathrm{~mm} \mathrm{~mm}^{-1} \mathrm{y}$ $0,289 \mathrm{~mm}$, respectivamente (figura 4), además la mayor parte de los valores promedio y de los valores de cada canaleta se ubican por debajo de la línea 1:1.

El valor promedio de la relación $T F / P_{g}$ fue de $82,1 \%$ y osciló entre 7,04 y 99,62\% para eventos entre 0,1 y 78,1 $\mathrm{mm}$. Se encontró además una correlación entre $T F / P_{g}$ y $P_{g}$ positiva $(\mathrm{r}=0,37)$ altamente significativa $(\mathrm{t}=5,90 \mathrm{gl}=$ $217, P<0,001)$. Sin embargo, esta relación mostró tener un comportamiento fuertemente no lineal (figura 5), descrito por la siguiente expresión:

$T F / P_{g} \%=\left(0,028+0,8 * P_{g} /\left(0,5065+P_{g}\right)\right) * 100 \quad[10]$

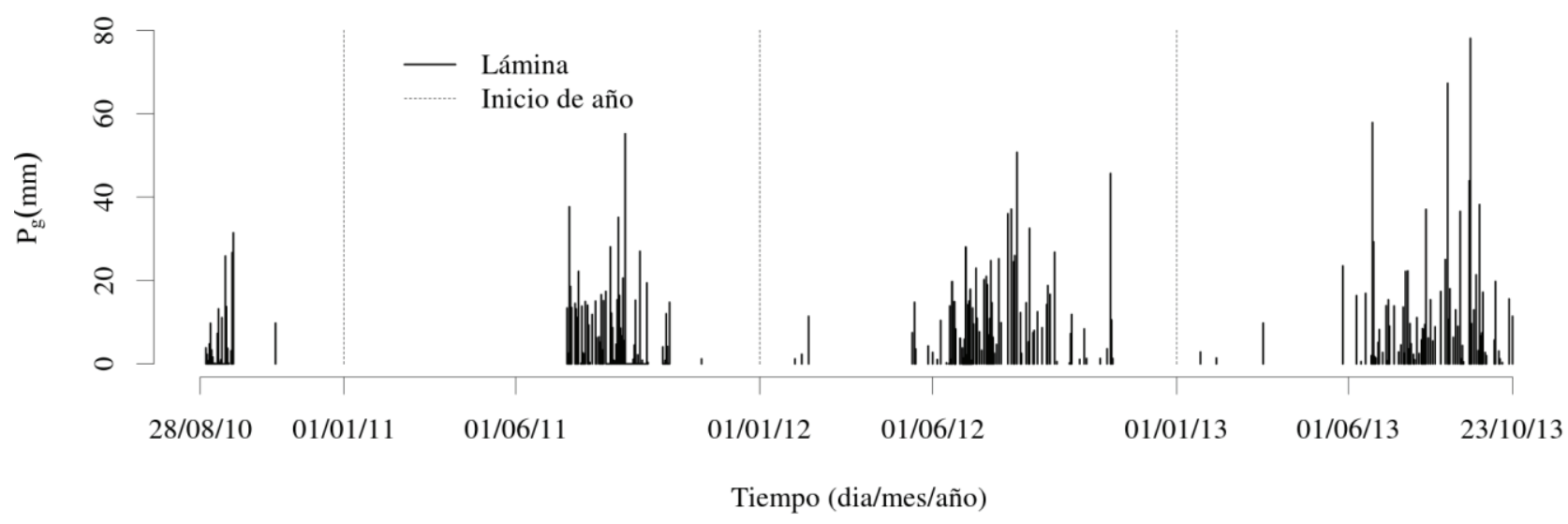

Figura 2. Registros diarios de lámina de precipitación bruta promedio $\left(P_{g}\right)$ para el periodo de estudio $(28 / 08 / 2010$ a 23/10/2013) en la estación ecohidrológica Alto Fresno.

Daily gross precipitation $\left(P_{g}\right)$ depth for the study period (08/28/2010 to 10/23/2013) at Alto Fresno ecohydrological research station. 

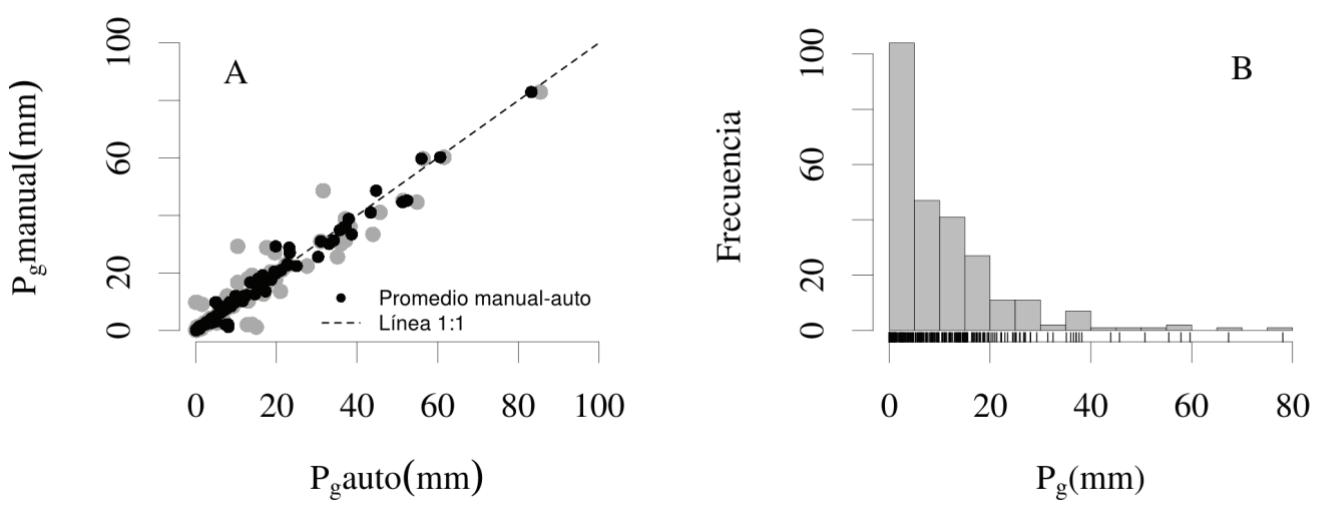

Figura 3. A) Relación entre lámina de precipitación bruta medida manual y automáticamente y el promedio de ambas mediciones. B) Distribución de frecuencias para lámina de precipitación bruta $\left(P_{g}\right)$ durante el periodo de estudio.

A) Daily gross precipitation $\left(P_{g}\right)$ depth measured manually, automatically and average between both measurements. B) Frequency distribution for daily gross precipitation $\left(P_{\mathrm{g}}\right)$ during the study period.

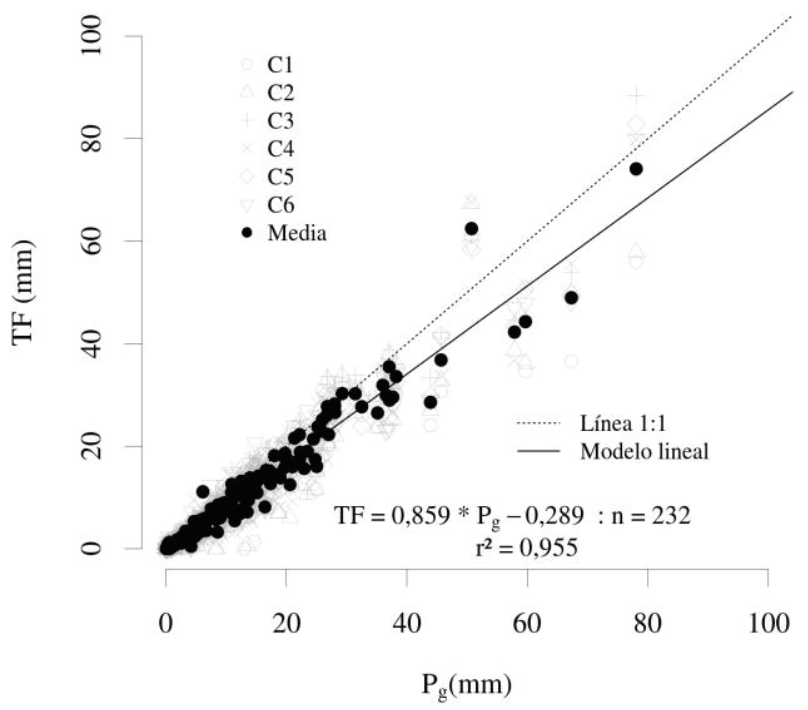

Figura 4. Gráfica de dispersión que muestra la lámina de precipitación bruta promedio $\left(P_{g}\right)$ versus precipitación directa $(T F)$ para las canaletas individuales ( $\mathrm{C} 1$ a $\mathrm{C} 6)$ y los valores promedio en un bosque de pino-encino de la estación ecohidrológica Alto-Fresno.

Scatterplot of gross precipitation depth $\left(P_{g}\right)$ versus throughfall depth $(T F)$ for individual troughs (C1 to $\mathrm{C} 6)$ and mean values.

Los datos muestran que durante los eventos pequeños la mayor parte de la precipitación se retiene en el dosel. La relación $T F / P_{g}$ se incrementa conforme aumenta la lámina de precipitación bruta. El modelo (ecuación 10) (figura 5) indica que es a partir de precipitaciones de aproximadamente $28 \mathrm{~mm}$ que la relación $T F / P_{g}$ alcanza un nivel relativamente estable con valores cercanos a $80 \%$.

Escorrentía cortical (SF). La escorrentía cortical mostró para todos los árboles una correlación positiva altamente significativa con la precipitación bruta $(P<0,001)$ y co-

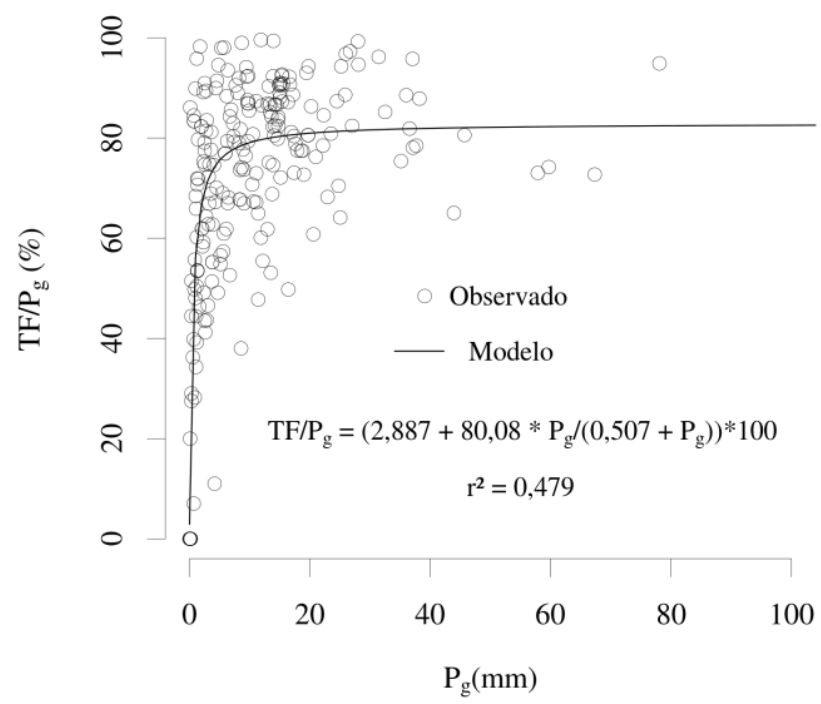

Figura 5. Precipitación directa $(T F)$ expresada como porcentaje de la precipitación bruta $\left(P_{g}\right)$ versus $P_{g}$ bajo bosque de pinoencino de la estación ecohidrológica Alto-Fresno.

Throughfall $(T F)$ expressed as a percentage of gross precipitation $\left(P_{g}\right)$ versus $P_{g}$ under pine-oak forest.

eficientes de correlación de Pearson mayores a 0,58 para todos los árboles estudiados.

La precipitación bruta y la escorrentía cortical presentaron un comportamiento lineal para todos los árboles (figura 6) tras alcanzar el valor de precipitación umbral necesario para generar escorrentía cortical (figura 6A). Los coeficientes de los modelos lineales (figura 6; ecuación 2) para cada árbol $(j)$ se presentan en el cuadro 3. Todos los modelos fueron altamente significativos para todos los árboles $(P<0,001)$. No se detectaron diferencias significativas para $p_{t}$ o $S_{t}$ entre $P$. leiophylla y $P$. devoniana. La 
$p_{t}$ promedio para todos los árboles presentó un valor de $0,0055 \pm 0,0102 \mathrm{~mm} \mathrm{~mm}^{-1}$, mientras que el $S_{t}$ promedio fue de $0,023 \pm 0,0392 \mathrm{~mm}$. Los datos del cuadro 2 ponen de manifiesto la heterogeneidad de estas dos variables en el bosque estudiado. En cuanto a pinos y encinos, las $p_{t}$ pro- medio fueron de 0,0058 $\pm 0,0123$ y $0,0048 \pm 0,0037 \mathrm{~mm}$ $\mathrm{mm}^{-1}$, respectivamente. Por otro lado, los encinos tuvieron una $S_{t}$ promedio ligeramente mayor que los pinos; 0,0205 $\pm 0,0465$ y $0,0286 \pm 0,0244 \mathrm{~mm}$ para pinos y encinos, respectivamente, sin haber diferencia significativa.
A

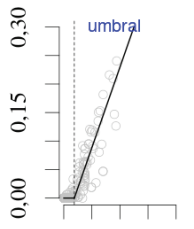

$\begin{array}{lll}0 & 40 \quad 80\end{array}$

P6

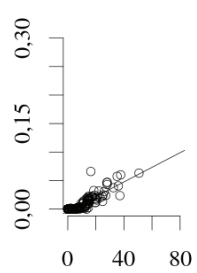

P12

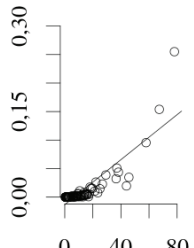

$\begin{array}{lll}0 & 40 \quad 80\end{array}$

Q3

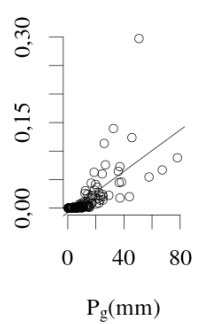

P1

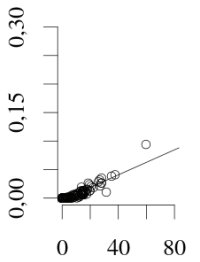

P7

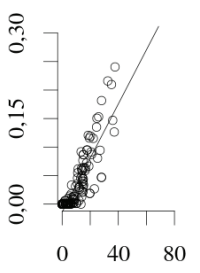

P13

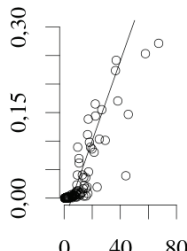

Q4

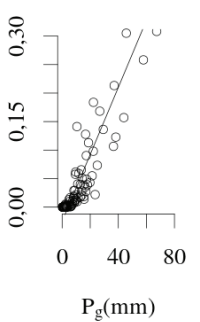

P2

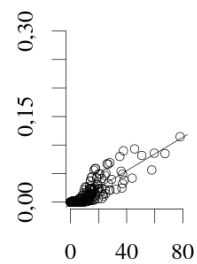

P8

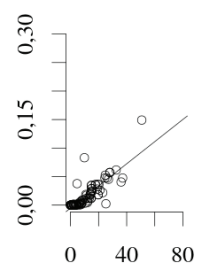

P14

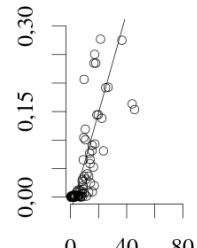

$0 \quad 40 \quad 80$

Q5

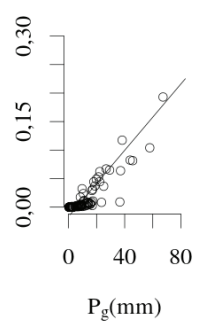

P3

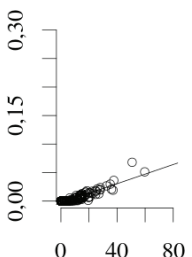

P9

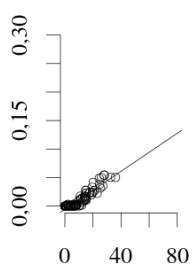

P15

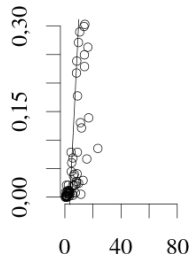

$0 \quad 40 \quad 80$

Q6

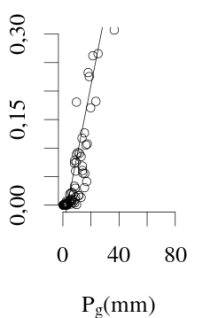

P4

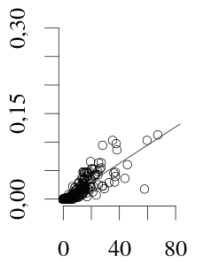

P10

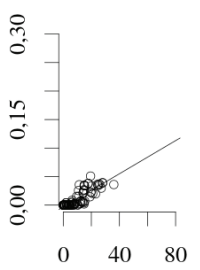

Q1

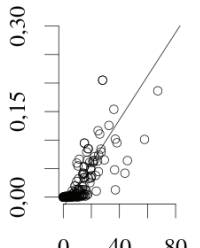

$0 \quad 40 \quad 80$

Q7

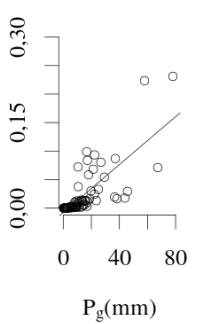

Figura 6. Gráficas de dispersión de precipitación bruta $\left(P_{g}\right)(\mathrm{mm}) v s$. lámina de escorrentía cortical $\left(S F_{L}\right)(\mathrm{mm})$ para cada árbol. $(\mathrm{A})$ Muestra la tendencia de los datos para uno de los árboles y las dos secciones, por debajo y arriba del umbral de generación de escorrentía cortical. En las gráficas (P1 - Q7) las líneas representan los modelos lineales obtenidos aplicando la ecuación [2]. Los encabezados corresponden a la nomenclatura de los árboles del cuadro 1.

Scatterplots of gross precipitation depth $\left(P_{g}\right)(\mathrm{mm})$ and stemflow depth $\left(S F_{L}\right)(\mathrm{mm})$ for the measured trees. (A) Shows the trend of the data for one of the trees and the two domains below and above the stemflow generation threshold. In graphs (P1-Q7) lines represent linear models for each tree according to eq. [2]. Graph headings correspond to tree names according to table 1. 
Cuadro 3. Parámetros de regresión lineal que describen la relación entre la escorrentía cortical $(S F)$ y precipitación bruta $\left(P_{g}\right)$ para árboles individuales de pino y encino en el bosque de la estación ecohidrológica Alto-Fresno.

Linear regression model parameters linking stemflow $(S F)$ to gross precipitation $\left(P_{g}\right)$ for individual pine and oak trees at Alto Fresno ecohydrological research station.

\begin{tabular}{|c|c|c|c|c|c|}
\hline Árbol & $p_{t}$ & $\begin{array}{c}S_{t} \\
(\mathrm{~mm})\end{array}$ & $\mathrm{r}^{2}$ & $\begin{array}{c}\text { REMC } \\
(\mathrm{mm})\end{array}$ & $n$ \\
\hline $\mathrm{P} 1$ & 0,0011 & 0,0034 & 0,828 & 0,005 & 127 \\
\hline $\mathrm{P} 2$ & 0,0015 & 0,0036 & 0,735 & 0,011 & 223 \\
\hline P3 & 0,0008 & 0,0032 & 0,825 & 0,004 & 133 \\
\hline P4 & 0,0016 & 0,0013 & 0,673 & 0,013 & 222 \\
\hline P5 & 0,0023 & 0,0011 & 0,724 & 0,013 & 131 \\
\hline P6 & 0,0013 & 0,0037 & 0,761 & 0,007 & 133 \\
\hline P7 & 0,0047 & 0,0141 & 0,747 & 0,025 & 131 \\
\hline P8 & 0,0019 & 0,0053 & 0,684 & 0,013 & 84 \\
\hline P9 & 0,0017 & 0,0057 & 0,862 & 0,006 & 82 \\
\hline P10 & 0,0015 & 0,0032 & 0,690 & 0,008 & 82 \\
\hline P11 & 0,0017 & 0,0042 & 0,790 & 0,007 & 81 \\
\hline P12 & 0,0020 & 0,0132 & 0,725 & 0,018 & 84 \\
\hline P13 & 0,0070 & 0,0390 & 0,633 & 0,077 & 89 \\
\hline P14 & 0,0088 & 0,0270 & 0,681 & 0,068 & 87 \\
\hline P15 & 0,0493 & 0,1788 & 0,758 & 0,246 & 82 \\
\hline Q1 & 0,0038 & 0,0173 & 0,612 & 0,038 & 174 \\
\hline Q2 & 0,0046 & 0,0149 & 0,737 & 0,030 & 171 \\
\hline Q3 & 0,0018 & 0,0790 & 0,472 & 0,026 & 121 \\
\hline Q4 & 0,0059 & 0,0252 & 0,801 & 0,042 & 90 \\
\hline Q5 & 0,0029 & 0,0167 & 0,759 & 0,024 & 90 \\
\hline Q6 & 0,0125 & 0,0395 & 0,737 & 0,066 & 85 \\
\hline Q7 & 0,0021 & 0,0076 & 0,581 & 0,026 & 89 \\
\hline
\end{tabular}

$\mathrm{r}^{2}$ : coeficiente de determinación. REMC: raíz cuadrada del error medio cuadrático del modelo lineal ecuación [2] en mm. $n$ : número de eventos.

En cuanto al índice de escurrimiento cortical, el valor promedio fue de $0,411 \pm 0,3 \mathrm{~L} \mathrm{~mm}^{-1}$. El análisis de varianza indicó la existencia de diferencia estadística entre encinos y pinos $(\mathrm{F}=5,63, \mathrm{gl}=1, P=0,0277)$, que presentaron valores promedio de $0,206 \pm 0,17$ y $0,506 \pm 0,31 \mathrm{~L} \mathrm{~mm}^{-1}$ respectivamente. En cuanto al volumen de almacenamiento del tronco, el promedio fue de 1,44 $\pm 1,16 \mathrm{~L}$ sin presentar diferencias entre pinos y encinos aunque el promedio de los pinos fue ligeramente mayor al de los encinos con $1,72 \pm 1,28$, y $0,84 \pm 0,51 \mathrm{~L}$, respectivamente.

Relación de la escorrentía cortical con variables dasométricas. En cuanto a las relaciones entre las variables daso- métricas y los coeficientes $p_{t}$ y $S_{t}$, no se encontraron correlaciones significativas. Sin embargo, se encontraron relaciones significativas entre las variables dasométricas del arbolado diámetro normal $(\mathrm{DN})$, altura total $(\mathrm{H})$ y superficie proyectada de copa (SC) y el índice de escorrentía cortical y el volumen de almacenamiento del tronco (figura 7). Los modelos lineales forzados al origen tuvieron coeficientes $\mathrm{r}^{2}>0,53$ y fueron significativos en todos los casos $(P \leq 0,05)$ (figura 7).

Los árboles $\mathrm{P} 7$ y Q6 presentaron valores de índice de escorrentía cortical y volumen de almacenamiento del tronco fuera de tendencia (figura 7). En general los coeficientes de determinación fueron más elevados para los modelos de los pinos que para los modelos de los encinos o con todos los árboles (pinos y encinos) (figura 7). Esto se atribuye a la cantidad de los árboles analizados para cada género, con valores de $\mathrm{n}=15 \mathrm{y} \mathrm{n}=7$ para pinos $\mathrm{y}$ encinos, respectivamente.

La variable dasométrica con mayor capacidad explicativa para el índice de escorrentía cortical fue el diámetro normal (DN) (figura 7). Esta variable explicó el 73,3, 62,2 y $70,1 \%$ de la variación en pinos, encinos y el total de los árboles, mientras que la altura (H) explicó entre $54,1 \%$ en encinos y el 73,1 \% en pinos. La superficie de copa (SC) fue la variable dasométrica con menor capacidad explicativa, entre 53,2 y $70,2 \%$.

Para el caso del volumen de almacenamiento del tronco, las variables dasométricas con mayor capacidad explicativa fueron, primero el diámetro normal que explicó el 81, 72,7 y $77,9 \%$ de su variación para pinos, encinos y todos los árboles, respectivamente, y segundo, la altura del árbol que explicó el 80, 67,9 y 79,2 \% de la variación para pinos, encinos y todos los árboles, respectivamente. La superficie de copa logró explicar solamente entre el 54,3 y el 63,9 \% de la variación del volumen de almacenamiento del tronco.

Para el caso del pino P7 (fuera de tendencia, ver figura 7) el valor de índice de escorrentía cortical fue de 1,38 L $\mathrm{mm}^{-1}$, mientras que el valor promedio para los pinos fue de $0,506 \mathrm{~L} \mathrm{~mm}^{-1} \mathrm{y}$ para todos los árboles de $0,411 \mathrm{~L} \mathrm{~mm}^{-}$ ${ }^{1}$. Esto significa que el P7 capta aproximadamente el 3,5 veces más que el promedio de los árboles. En el caso del encino fuera de tendencia (Q6, ver figura 7), presentó un índice de escorrentía cortical de $0,578 \mathrm{~L} \mathrm{~mm}^{-1}$ mientras que para el promedio de los encinos fue de $0,21 \mathrm{~L} \mathrm{~mm}^{-1}$; valor que representa 2,75 veces más que el promedio de los encinos o 1,4 veces más que el promedio de todos los árboles.

La observación directa en campo reveló que el árbol P7 posee una copa con arquitectura atípica, con una bifurcación del tronco principal aproximadamente a los $4,50 \mathrm{~m}$ de altura, mientras que el árbol Q6 presentó una bifurcación del tronco principal a los 2,3 $\mathrm{m}$ y bifurcaciones de los troncos secundarios a los 3,5 y 4,2 m. La relevancia de estas observaciones en la generación de escorrentía cortical, índice de escorrentía cortical y volumen de almacenamiento del tronco será abordado posteriormente en la discusión. 
Interceptación (I). Se ubicó entre 0,01 y 18,4 mm con una media de 2,2 $\pm 2,7 \mathrm{~mm}$. La relación entre interceptación y precipitación bruta mostró un comportamiento lineal $\left(\mathrm{r}^{2}=0,572, P<0,001\right.$; figura 8$)$ descrito por la siguiente expresión:

$$
I=0,1701 P_{g}+0,2921
$$

Por otro lado, la relación entre la proporción de pérdida por interceptación como función de la precipitación bruta tuvo un comportamiento de decaimiento hiperbólico (figura 9, ecuación 12). Esta relación indica que el mayor porcentaje de interceptación ocurre en eventos pequeños $(<5 \mathrm{~mm})$ pero tiende a estabilizarse en aproximadamente $20 \%$ para precipitación bruta $>20 \mathrm{~mm}$ (figura 9 ).

$$
I \%=17,033+\left(40,563 /\left(P_{g}+0,507\right)\right)
$$

Partición de la precipitación. La interceptación (I) se estimó en 19,4 \%, mientras que la proporción de precipitación como escorrentía cortical $(S F)$ fue $2,4 \%$. Por lo que la pérdida por interceptación $\left(E_{i}\right)$ fue de $495,7 \mathrm{~mm}$, que corresponde a $17,2 \%$. Mientras que la precipitación neta $\left(P_{n}\right)$ fue de $83,0 \%$ (2.400,5 mm) (cuadro 4$)$.

\section{DISCUSIÓN}

Interceptación. El porcentaje de interceptación $(I=19,4$ \%) encontrado en el presente estudio se ubica dentro del rango de valores reportados en la literatura especializada en bosques similares. Navar et al. (2013) reportan $16,6 \%$ de interceptación para un bosque de pino-encino de Nuevo León, México. Mientras que Cantú y González (2002) reportan valores de 19,2, 13,6 y 23,0 \% en bosques de pino, encino y pino-encino (NE México) con diámetros norma-
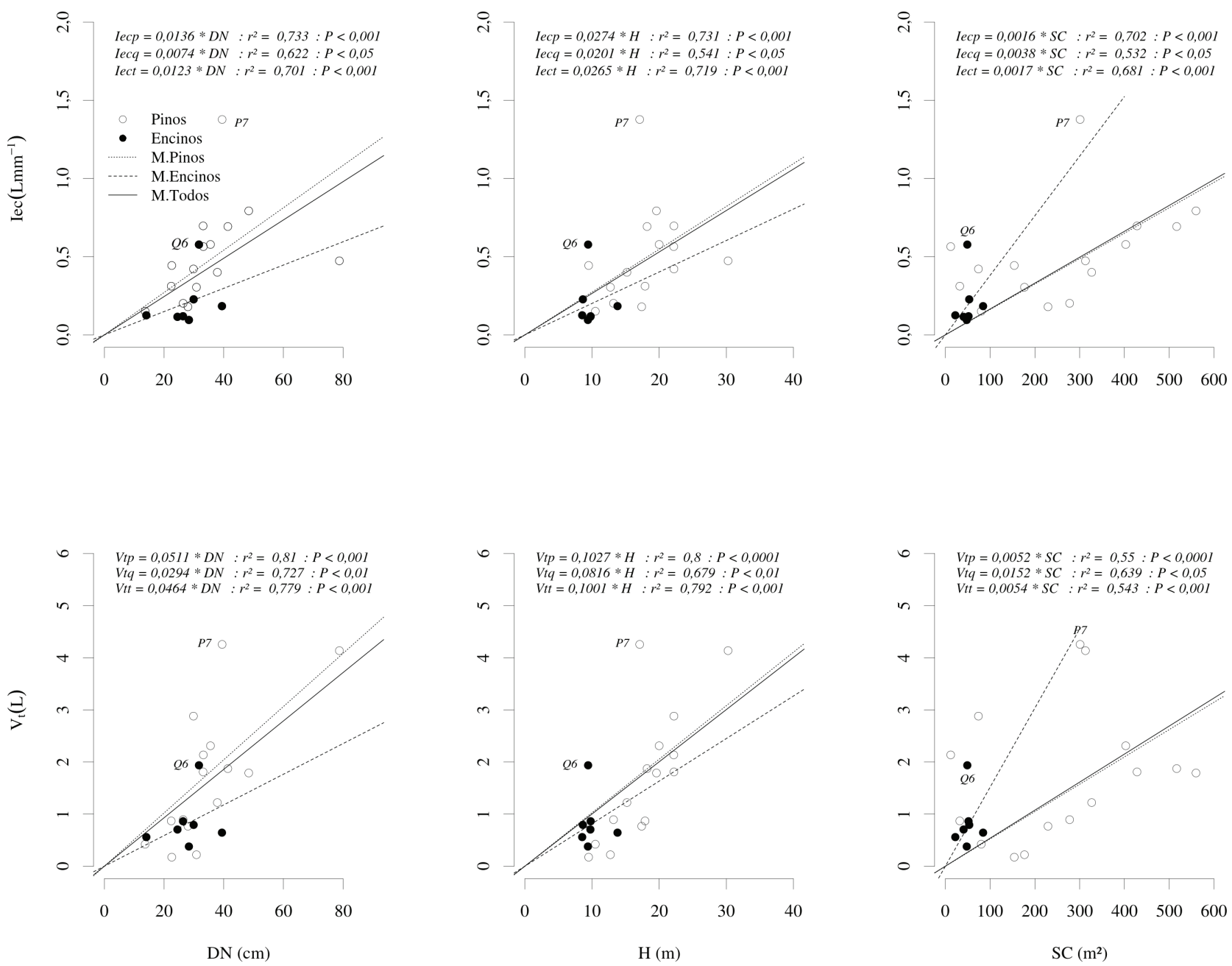

Figura 7. Relaciones entre el Iec y $V_{t}$ y las variables dasométricas del arbolado (DN) diámetro normal, (H) altura total, (SC) superficie de copa para pinos (Iecp, Vtp), encinos (Iecq, Vtq) y el total de árboles (Iect, Vtt). Nótese el árbol fuera de tendencia (P7).

Scatterplots between $I e c$ and $V_{t}$ and tree characteristics variables (DN) diameter at breast height, $(\mathrm{H})$ total height and (SC) crown surface for pines (Iecp, Vtp), oaks (Iecq, Vtq) and whole of measured trees (Iect, Vtt). Note the outlier tree (P7). 


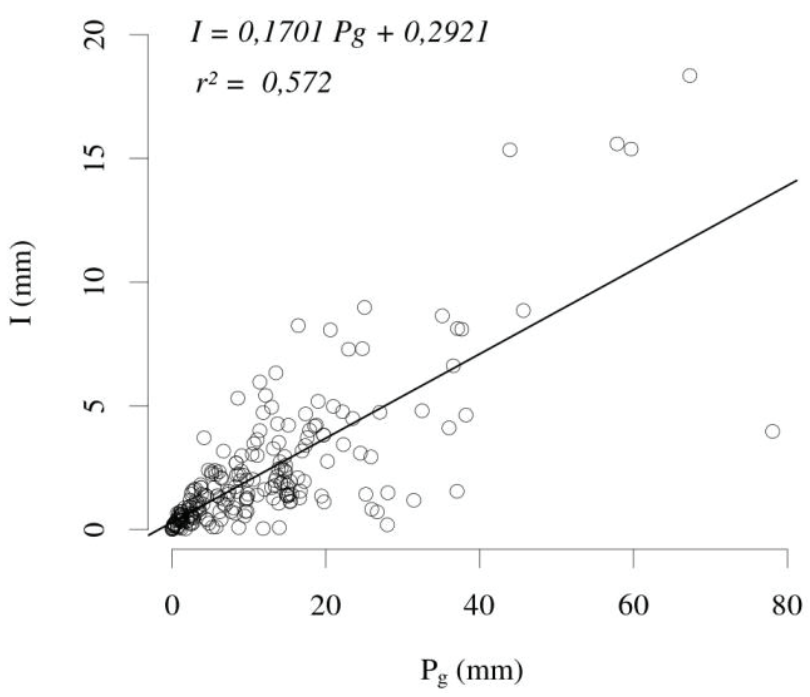

Figura 8. Lámina de agua interceptada por el dosel $(I)(\mathrm{mm})$ en función de la precipitación bruta $\left(P_{g}\right)(\mathrm{mm})$ en bosque de pinoencino.

Water depth intercepted by the canopy $(I)(\mathrm{mm})$ as a function of gross precipitation $\left(P_{g}\right)(\mathrm{mm})$ for the pine-oak forest.

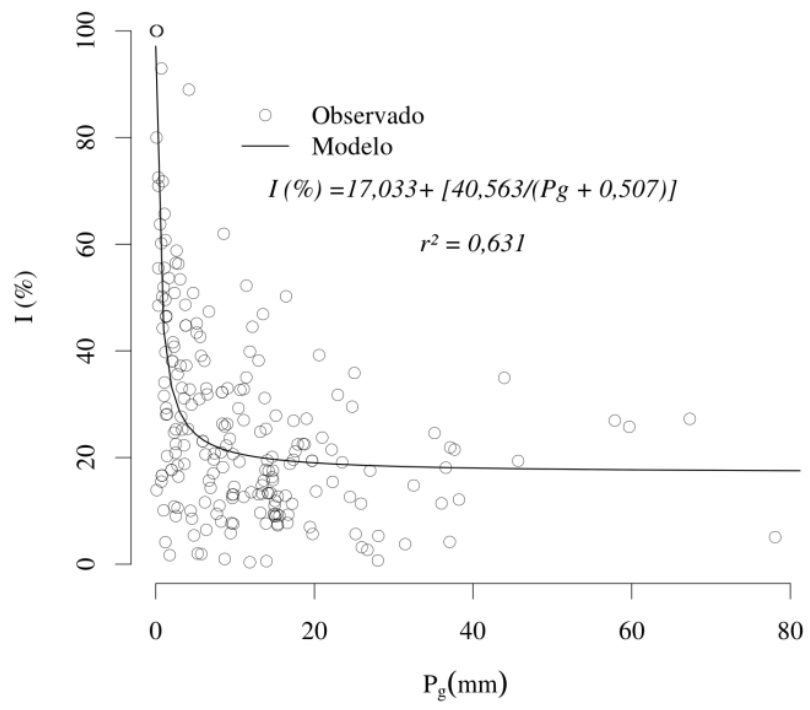

Figura 9. Proporción de interceptación $(I \%)$ en función de la precipitación bruta $\left(P_{g}\right)$ en bosque de pino-encino.

Canopy interception $(I \%)$ proportion as a function of gross precipitation $\left(P_{g}\right)$ for the pine-oak forest.

les promedio de 32,16 y $34 \mathrm{~cm}$, alturas $12,0,9,0$ y $16,4 \mathrm{~m}$ y superficies de copa 37,2, 36,8 y 77,8 $\mathrm{m}^{2}$ respectivamente. Mientras que Carlyle-Moses y Price (2007) reportan 15,8 $\%$ de interceptación en bosque de pino-encino en el NE de México con mayor densidad de individuos (819 árboles $\left.\mathrm{ha}^{-1}\right)$ pero menor área basal $\left(31,3 \mathrm{~m}^{2} \mathrm{ha}^{-1}\right)$ que el sitio del presente estudio $\left(50,7 \mathrm{~m}^{2} \mathrm{ha}^{-1}\right)$. Los resultados indican que
Cuadro 4. Componentes de la partición de la precipitación en el bosque de pino-encino de la estación ecohidrológica Alto-Fresno.

Precipitation partition components for the pine-oak forest at Alto Fresno ecohydrological research station.

\begin{tabular}{cccc}
\hline Componente & $\begin{array}{c}\text { Lámina } \\
(\mathrm{mm})\end{array}$ & $\begin{array}{c}\text { Error estándar } \\
\text { de la media }\end{array}$ & $\begin{array}{c}\text { Proporción } \\
(\%)\end{array}$ \\
\hline$P_{g}$ & 2881,8 & 0,76 & 100,0 \\
$P_{n}$ & 2400,5 & - & 83,0 \\
$T F$ & 2322,7 & 0,66 & 80,6 \\
$I$ & 559,1 & 0,18 & 19,4 \\
$E_{i}$ & 495,7 & - & 17,2 \\
$S F$ & 69,5 & 0,048 & 2,4 \\
\hline
\end{tabular}

$P_{g}$ : precipitación bruta, $P_{n}$ : precipitación neta, $T F$ : precipitación directa, $I$ : onterceptación, $E_{i}$ : pérdida por interceptación, $S F$ : escorrentía cortical.

la proporción de precipitación bruta interceptada por el dosel arbóreo es mayor para eventos pequeños y menor en los grandes. Este patrón concuerda con lo mencionado en investigaciones previas para distintos ambientes (CarlyleMoses 2004, Návar 2011). En el sitio del presente estudio tal interceptación tiende a estabilizarse una vez alcanzado un umbral de precipitación bruta $\sim 20 \mathrm{~mm}$ (figura 9) y este comportamiento concuerda con lo reportado por CarlyleMoses (2004) y Flores et al. (2013).

El modelo empírico propuesto para representar la proporción de interceptación como función de la precipitación bruta $(I \%=f(P))$ (ecuación 12) representa en forma adecuada el proceso aún para eventos extremos como el del 16 de septiembre de 2013 (figura 9) que presenta un periodo de retorno estimado de nueve años para la estación 16055 "Jesús del Monte" y de 17 años para la estación 16001 "Acuitzio del Canje"3.

Ya que las mediciones en el presente estudio fueron cada 24 horas, los datos obtenidos no permiten establecer una relación entre la intensidad de la precipitación y la partición de esta. Para este tipo de análisis se requiere contar con datos en intervalos de tiempo del orden de horas o minutos como en los realizados por Holwerda et al. (2010) empleando equipos automatizados. Sin embargo, esto se encuentra por ahora fuera del alcance de este trabajo.

Escorrentía cortical. Actualmente se sabe que el agua concentrada como escorrentía cortical puede ser una fuente de agua importante para la vegetación, especialmente en ambientes áridos y semiáridos (Náver y Bryan, 1990, Martinez-Meza y Whitford 1996, Johnson y Lehmann 2006).

\footnotetext{
Periodos de retorno calculados empleando la base de datos de las estaciones climáticas del Sistema Meteorológico Nacional (SMN, 2014ab).
} 
La escorrentía cortical concentra la precipitación interceptada por el dosel en un área pequeña (Johnson y Lehmann 2006) y puede favorecer la infiltración preferencial (Llorens y Domingo 2007).

Johnson y Lehman (2006) reportaron porcentajes de escorrentía cortical muy variados para distintos tipos de ecosistemas y especies estudiadas; entre 0,07 y $22,0 \%$ (Johnson y Lehman 2006). En el caso de México, Holwerda et al. (2010) reportaron escorrentía cortical de 4,0 \% de la precipitación bruta en un bosque de niebla secundario y Charles (1998) reportó valores de escorrentía cortical de $0,24,0,74$ y $0,71 \%$ en bosques de pino, encino y pinoencino respectivamente del norte de México, mientras que Cantú y González (2001) reportaron valores de 0,60, 0,50 y $0,03 \%$ en bosques de pino, encino y pino-encino del NE de México (Nuevo León). La proporción de escorrentía cortical $(2,4 \%)$ en este estudio se encuentra por encima de los valores reportados para bosques similares, pero por debajo de los reportados para bosque de niebla.

El valor inesperado de 2,4\% de escorrentía cortical en este estudio puede deberse a dos factores. Primero, a la densidad del arbolado (677,8 individuos ha- ${ }^{-1}$ ), mayor que en sitios similares estudiados previamente y segundo, a la edad promedio de los árboles, aproximadamente 18 años. La morfología de copa, en especial el ángulo de las ramas está estrechamente relacionado con la edad y la densidad del arbolado; los árboles jóvenes presentan ramas con ángulos más elevados respecto a la horizontal (Mäkkinen y Colin 1998). Previamente se ha reportado un efecto positivo de la inclinación de las ramas sobre la escorrentía cortical en bosques tropicales secos, de eucalipto y pino (Crockford y Richardson 1990). Steinbuck (2002) reportó para bosques de coníferas en el NE de Estados Unidos que la cantidad de agua de escurrimiento cortical está determinada por el "hábito" de las ramas 4 , el área proyectada de copa y la superficie del tronco. De forma que los árboles con la mayor cantidad de ramas inclinadas positivamente (arriba de la horizontal), pero con la mayor área proyectada de copa y troncos más lisos son los árboles con mayor producción de escorrentía cortical.

Respecto a la morfología de copa de los árboles en la estación ecohidrológica Alto-Fresno Santiago-Bedolla (en proceso) menciona que el $82,3 \%$ de las ramas de 14 pinos muestreados presentan ángulos positivos. Por otro lado, la inspección de campo mostró que el árbol P7 se bifurca a los 4,5 m de altura. Algo similar ocurrió con el árbol Q6 con bifurcaciones del tronco principal y secundarios a los 2,3, 3,5 y 4,2 m, respectivamente. Tanto P7 como Q6 presentaron una gran cantidad de ramas con hábito positivo (sensu Steinbuck 2002). Q6 fue el encino con mayor pendiente en la relación de precipitación bruta $v S S F_{L}$ (figura 6). Es importante recordar que los árboles Q6 y P7 estu-

\footnotetext{
Steinbuck (2002) define como branching habit o hábito de las ramas a la proporción de ramas positivas y negativas respecto a la horizontal en la copa del árbol (p. 18)
}

vieron fuera de tendencia en cuanto a las relaciones entre variables dasométricas y índice de escorrentía cortical y volumen de almacenamiento del tronco (figura 7).

Debido a que en el presente estudio la selección de los árboles para cuantificación de la escorrentía cortical se realizó completamente al azar, el hecho que solo uno de los 22 árboles analizados haya estado fuera de tendencia lo atribuimos a la distribución probabilística de la morfología de copa y el carácter aleatorio del muestro.

Índice de escorrentía cortical. Este índice estuvo correlacionado significativamente con el diámetro normal, la altura del árbol y la superficie de copa (figura 7). Sin embargo, los factores que modifican o controlan la escorrentía cortical influirán en el índice de escorrentía cortical. En el presente trabajo dicho índice para el árbol (P7) llegó a ser hasta 3,35 veces más alto que el promedio de los árboles y se explicó por la morfología de copa atípica.

El efecto de concentración de humedad del suelo asociado a la escorrentía cortical ha sido reportado en vegetación arbustiva (Zhang et al. 2013) y en vegetación arbórea (Ladekarl 1998) y se ha postulado que la escorrentía cortical que ingresa al suelo alrededor de la base de un árbol es un mecanismo importante de resistencia a la sequía (Martinez-Meza y Whitford 1996). Guan et al. (2010) demostraron que en suelos arcillosos y bajo ciertas condiciones, la presencia de raíces de árboles favorece la recarga del suelo y los mantos freáticos, posibilitando una percolación hasta del $20 \%$ de la precipitación anual, mientras que sin presencia de este tipo de vías preferenciales de infiltración la percolación es $<1 \%$. Si bien la determinación de los procesos de infiltración, recarga edáfica y de acuíferos se encuentra fuera del alcance de este trabajo, de acuerdo con las condiciones del suelo en el área de estudio, es posible que mecanismos similares a los descritos por Guan et al. (2010) estén ocurriendo. Los resultados ponen de manifiesto la necesidad de evaluar el aporte del índice de escorrentía cortical y escorrentía cortical en el balance hídrico a escala local, de cuenca y su posible influencia en la recarga edáfica y de acuíferos, como ha sido descrito previamente por varios investigadores (Návar 2011).

Consideraciones finales. Con base en lo anteriormente expuesto, se propone que investigaciones futuras se centren en los siguientes temas: a) Relación de la morfología y arquitectura de copa con la escorrentía cortical y la captación hídrica, en especial en árboles con morfologías atípicas. Además las morfologías atípicas deberán de describirse y tipificarse para las diferentes especies arbóreas. b) Relación entre escorrentía cortical e infiltración preferencial en distintas especies y condiciones de suelo. c) Balances hídricos locales incluyendo las pérdidas por interceptación y el aporte del índice de escorrentía cortical del dosel. d) Cuantificar y caracterizar el aporte de niebla en la precipitación total, empleando métodos directos, como redes de niebla. 


\section{CONCLUSIONES}

La proporción de precipitación interceptada se encuentra dentro de los rangos reportados en la literatura para bosques similares, sin embargo, la proporción de escorrentía cortical en el área de estudio fue mayor que la reportada previamente para bosques de pino-encino similares en el noreste de México $(<1 \%)$. Esta diferencia se atribuye a tres causas: la elevada densidad de árboles, la composición del arbolado 66,7 \% Pinus spp. y la edad promedio (18 años). Esta última determina la inclinación positiva de las ramas.

El índice de escorrentía cortical (Iec) y el volumen de agua retenido por el árbol $(V)$ son explicados hasta un 73 y $80 \%$ por variables dasométricas como la altura y el diámetro normal, respectivamente. Por lo que estas variables pueden funcionar como predictores eficientes de tal índice y el volumen de almacenamiento del tronco. Además, en el presente trabajo se detectaron dos árboles fuera de tendencia (P7 y Q6), cuyos valores de índice de escorrentía cortical y volumen de almacenamiento del tronco fueron más elevados que el resto de los pinos el primero y los encinos el segundo. Este comportamiento se relacionó con copas bifurcadas y con gran cantidad de ramas con ángulos positivos en ambos árboles. Estos resultados ponen de manifiesto la necesidad de conocer la relación entre morfología y estructura de copa a un nivel más detallado para poder comprender y modelar con mayor eficiencia diversos componentes de la partición de la precipitación, en especial la escorrentía cortical.

\section{AGRADECIMIENTOS}

Los autores deseamos agradecer a los cuatro árbitros anónimos que ayudaron a mejorar significativamente este trabajo. También deseamos agradecer a las siguientes personas: Rafael Morales Chávez, Ana Isabel Santiago Bedolla, Edgar Mora Damián, Dulce María Rosas Rangel, Carolina Adriana Ruiz Díaz y Rosa Leiz Ponce Díaz por la ayuda durante la construcción e instalación del equipo, la obtención de variables dasométricas y las mediciones en campo. También a Yolanda Chávez H. por el apoyo en la colecta de datos de campo, asimismo a Zeus, Froya, Kira, Tecutli y Sasa por la vigilancia y resguardo del instrumental. El financiamiento para esta investigación fue proporcionado por los proyectos SEP-PROMEP-23-005-UMSNH "Variación espacial de la infiltración superficial y sus propiedades de control en el sur de la Cuenca de Cuitzeo", CIC-UMSNH 2011-2012 "Caracterización detallada del funcionamiento ecohidrológico para la captación hídrica, en una microcuenca de Cuitzeo" y CIC-UMSNH 2013 "Cartografía de permeabilidad edáfica empleando métodos geoestadísticos a escala de cuenca".

\section{REFERENCIAS}

Calder IR, CHR Kidd. 1978. A note on the dynamic calibration of tipping-bucket gauges. Journal of Hydrology 39(3-4):
383-386.

Cantú S I, H González. 2001. Interception loss, throughfall and stemflow chemistry in pine and oak forests in northeastern Mexico. Tree Physiology 21: 1009-1013.

Cantú S I, H González. 2002. Propiedades hidrológicas del dosel de los bosques de pino-encino en el noreste de México. Ciencia UANL 5(1): 72-77.

Cantú S I, H González. 2005. Pérdidas por intercepción de la lluvia en tres especies de matorral submontano. Ciencia UANL 8(1): 80-85.

Carlyle-Moses DE. 2004. Throughfall, stemflow, and canopy interception loss fluxes in a semi-arid Sierra Madre Oriental matorral community. Journal of Arid Environments 58(2): 181-202.

Carlyle-Moses DE, AG Price. 2007. Modelling canopy interception loss from a Madrean pine-oak stand, Northeastern Mexico. Hydrological Processes 21(19): 2572-2580.

Charles MF. 1998. Medición y estimación de los componentes de la intercepción en cuatro comunidades representativas del nordeste de México. Tesis de Maestría en Ciencias Forestales. Monterrey, México. Universidad Autónoma de Nuevo León. 82 p.

Crockford RH, DP Richardson 1990. Partitioning of rainfall in a eucalypt forest and pine plantation in southeastern Australia. II: Stemflow and factors affecting stemflow in a dry sclerophyll eucalypt forest and Pinus radiata plantation. Hydrological Processes 4: 145-155.

David JS, F Valente, JHC Gash. 2005. Evaporation of intercepted rainfall. In Anderson MG ed. Encyclopedia of Hydrological Sciences Volume 1. Chichester, England. John Wiley. p. 627-634.

Díaz-Fernández E, AF Gómez-Tagle. 2012. Intercepción pluvial en distintos ecosistemas de la cuenca hidrográfica de Cointzio, Michoacán. In Bravo M, G Barrera, ME Mendoza, JT Sánez, F Bahena, R Sánchez eds. Contribuciones para el desarrollo sostenible de la cuenca de Cuitzeo, Michoacán. Michoacán, México. UNAM Centro de Investigaciones en Geografía Ambiental. p. 105-112.

Flores AE, F Becerra, E Buendía, F Carrillo, G H Terrzas, T Pineda, M Acosta. 2013. Intercepción de lluvia por matorral inerme espinoso en Atotonilco el Grande, Hidalgo. Revista Mexicana de Ciencias Agrícolas 4(2): 285-298.

FAO (Food and Agriculture Organization, IT). 2011. State of the World's Forests. Rome, Italy. FAO. 164 p.

Garduño-Monroy VH, N Giordano, JA Ávila-Olivera, VM Hernández-Madrigal, A. Sámano-Nateras, JE Díaz-Salmerón. 2014. Estudio hidrogeológico del sistema acuífero de Morelia, Michoacán, para una correcta planificación del territorio. In Vieyra A, A Larrazabal eds. Urbanización, Sociedad y Ambiente. Michoacán, México. UNAM Centro de Investigaciones en Geografía Ambiental. p. 197-222.

Gash JHC, AJ Morton. 1978. An application of the Rutter model to the estimation of the interception loss from Thetford forest. Journal of Hydrology 38: 49-58.

Gash JHC, IR Wright, CR Lloyd. 1980. Comparative estimates of interception loss from three coniferous forests in Great Britain. Journal of Hydrology 48(1-2): 89-105.

Gómez-Tagle A. 2008. Variabilidad de las propiedades edáficas relacionadas con la infiltración y conductividad hidráulica superficial en la cuenca de Cuitzeo. Tesis doctoral en Ciencias Biológicas. Michoacán, México. Universidad Michoa- 
cana de San Nicolás de Hidalgo. 164 p.

Guan H, J Simunek, BD Newman, JL Wilson. 2010. Modelling investigation of water partitioning at a semiarid ponderosa pine hillslope. Hydrological Processess 24(1): 1095-1105.

Holwerda F, FN Scatena, LA Bruijnzeel. 2006. Throughfall in a Puerto Rico lower montane rain forest: A comparison of sampling strategies. Journal of Hydrology 327(1): 592-602.

Holwerda F, LA Bruijnzeel, LE Muñoz-Villers, M Equihua, H Asbjornsen. 2010. Rainfall and cloud water interception in mature and secondary lower montane cloud forests of central Veracruz, Mexico. Journal of Hydrology 384(1): 84-96.

Holwerda F, LA Bruijnzeel, VL Barradas, J Cervantes. 2013. The water and energy exchange of a shaded coffee plantation in the lower montane cloud forest zone of central Veracruz, Mexico. Agricultural and Forest Meteorology 173(1): 1-13.

Hölscher D, L Kohler, AIJM van-Dijk, LA Bruijnzeel. 2004. The importance of epiphytes to total rainfall interception by a tropical montane rainforest in Costa Rica. Journal of Hydrology 292(1): 308-322.

INEGI (Instituto Nacional de Estadística y Geografía, MX). 2013. Censo de población y vivienda 2010. INEGI; Disponible en: http://www.inegi.org.mx/sistemas/olap/proyectos/ bd/consulta.asp? $\mathrm{p}=17159 \& \mathrm{c}=17547 \& \mathrm{~s}=$ est.

Johnson MS, J Lehmann. 2006. Double-funneling of trees: Stemflow and root-induced preferential flow. Ecoscience 13 (3): 324-333.

Ladekarl UL. 1998. Estimation of the components of soil water balance in a Danish oak stand from measurements of soil using TDR. Forest Ecology and Management 104(1): 227-238.

Leyton L, ERC Reynolds, FB Thompson. 1967. Rainfall interception in forest and moorland. In Sopper WE, HW Lull eds. Int. Syrup. on Forest Hydrology. Oxford, UK. Pergamon Press. p. 163-178.

Levia DF Jr, EE Frost. 2003. A review and evaluation of stemflow literature in the hydrologic and biogeochemical cycles of forested and agricultural ecosystems. Journal of Hydrology 274 (1-4): 1-29.

Llorens P, F. Domingo. 2007. Rainfall partitioning by vegetation under Mediterranean conditions. A review of studies in $\mathrm{Eu}-$ rope. Journal of Hydrology 335: 37-54.

Martínez-Meza E, WG Whitford. 1996. Stemflow, throughfall and channelization of stemflow by roots in three Chihuahuan desert shrubs. Journal of Arid Environments 32(1): 271-287.

Mäkinen H, Colin F. 1998. Predicting branch angle and branch diameter of Scots pine from usual tree measurements and stand structural information. Canadian Journal of Forest Research 28(11): 1686-1696.

Návar J, F Carlyle-Moses, E Martínez. 1999. Interception loss from the tamaulipan matorral thornscrub of northeastern
Mexico: an application of the Gash analytical interception loss model. Journal of Arid Environments (40): 1-10.

Návar J. 2011. Stemflow variation in Mexico's northeastern forest communities: Its contribution to soil moisture content and aquifer recharge. Journal of Hydrology 408 (1-2): 35-42.

Nauelhual L, P Donoso, A Lara, D Núñez, C Oyarzún, E Neira. 2007. Valuing ecosystem services of chilean temperate rainforests. Environment, Development and Sustainability (9): 481-499.

Peñuela-Arévalo LA, JJ Carrillo-Rivera. 2013. Definición de zonas de recarga y descarga de agua subterránea a partir de indicadores superficiales centro-sur de la Mesa Central, México. Investigaciones Geográficas, Boletín del Instituto de Geografía, UNAM 81: 18-32.

R Core Team (2014). R: A language and environment for statistical computing. R Foundation for Statistical Computing, Vienna, Austria. Disponible en http:/www.R-project.org/

Rutter AJ, AJ Morton, PC Robins. 1975. A predictive model of rainfall interception in forests. II. Generalization of the model and comparison with observations in some coniferous and hardwood stands. The Journal of Applied Ecology 12(1): 367-380.

Rzedowski J. 2006. Vegetación de México. Distrito Federal, México. Comisión Nacional para el Conocimiento y Uso de la Biodiversidad. 504 p.

SMN (Servicio Meteorológico Nacional, MX). 2014a. Climatología estadística, estación 16055 Jesús del Monte, Mich. Disponible en http://smn.cna.gob.mx/climatologia/Diarios/16055.txt

SMN (Servicio Meteorológico Nacional, MX). 2014b. Climatología estadística, estación 16001 Acuitzio del Canje, Mich. Disponible en http://smn.cna.gob.mx/climatologia/Diarios/16001.txt

SMN (Servicio Meteorológico Nacional, MX). 2011a. Normales climatológicas, periodo 1951-2010; estación 16055 Jesús del Monte, Michoacán de Ocampo. Consultado 20 nov. 2013. Disponible en http://smn.cna.gob.mx/climatologia/ Normales5110/NORMAL16055.TXT

SMN (Servicio Meteorológico Nacional, MX). 2011b. Normales climatológicas, periodo 1951-2010; estación 16001 Acuitzio del Canje, Michoacán de Ocampo. Consultado 20 nov. 2013. Dispoible en http://smn.cna.gob.mx/climatologia/Normales5110/NORMAL16001.TXT

Steinbuck E. 2002. The influence of tree morphology on stemflow in a redwood region second-growth forest. Ms.C. Thesis in Geosciences. California, United States. California State University. 55 p.

Zhang Y, X Wang, R Hu, Y Pan, H Zhang. 2013. Stemflow in two xerophytic shrubs and its significance to soil water and nutrient enrichment. Ecological Reserach 28(4): 567-569. 